\title{
VITTORIO EMANUELE ORLANDO: STUDIOSO, POLITICO, COSTITUENTE
}

\author{
Nota del m.e. VALERIO ONIDA (*)
}

(Adunanza del 16 aprile 2015)

\begin{abstract}
SuNTO. - Lo scritto ripercorre sommariamente la carriera di Vittorio Emanuele Orlando, studioso precoce e presto divenuto maestro del diritto pubblico, e protagonista politico nell'epoca prefascista, soffermandosi soprattutto sul ruolo da lui svolto all'Assemblea Costituente, di cui era il decano. Si ricordano i suoi importanti interventi, fra cui quello contro la ratifica del Trattato di pace, e quelli particolarmente approfonditi e appassionati sul progetto di Costituzione. In essi Orlando palesava le sue riserve sulle scelte fatte dalla maggioranza dell'Assemblea in tema di forma di governo, ma manifestava infine il suo augurio fiducioso per il futuro del Paese e la sua convinzione che si fosse allora, nel mondo, all'inizio di una nuova era, in cui si sarebbe affermato un nuovo "tipo di Stato", di fronte al quale anche l'Italia, in condizioni di parità con gli altri Stati, avrebbe dovuto rinunciare "all'orgogliosa affermazione della sovranità assoluta".
\end{abstract}

$* * *$

ABSTRACT. - The article traces the career of Vittorio Emanuele Orlando: early scholar, he soon became a master in public law, and a political protagonist before the advent of the fascist regime, to which he never gave his adhesion, focusing mainly on his role in the Constituent Assembly, of which he was the dean. His important interventions are recalled, including the one against the ratification of the Peace Treaty, and those, particularly deepened and passionate, on the draft of the Constitution. Orlando expressed his reservations about the choices made by the majority of the Assembly about the form of government, but he finally expressed his hope in the future of the country and his belief in the beginning of a new worldwide era, in which a new "type of State" would take place, in which Italy too, at par with the other States, would have to abandon "the proud affirmation of the absolute sovereignty".

(") Università degli Studi di Milano, Italia. E-mail: valerio.onida@onidalex.it 


\section{ORLANDO STUDIOSO PRECOCE E MAESTRO DEL DIRITTO}

Vittorio Emanuele Orlando è stato una persona straordinaria, che ha attraversato da protagonista due secoli.

Anzitutto come studioso e giurista. Quando nel 1881 - lo stesso anno in cui si laurea - vince il "Premio ordinario di Fondazione Pizzamiglio", assegnato dall'Istituto Lombardo di Scienze e Lettere, classe di lettere e scienze morali e politiche, con un saggio su "La riforma elettorale", ${ }^{1}$ Orlando ha 21 anni.

La Commissione giudicatrice del premio $^{2}$ non ne conosceva il nome, ma sapeva che "l'autore è assai giovane" (l'indizio era il fatto che egli "dovette compiere gli studi universitari nel 1879"). Scriveva così: "In un giovane appena uscito dagli studi ... è raro in condizioni tali ritrovare tanta assennatezza, congiunta con una coltura certo non comune". ${ }^{3}$

Il giovane premiato diventerà il fondatore di una Scuola del diritto pubblico, aperto alle culture giuridiche di altri paesi, come Germania, Francia e Inghilterra, di cui conosceva non solo la dottrina, ma anche la storia e le prassi.

A 29 anni pubblica i Principi di diritto costituzionale, la cui prima edizione è del 1889. ${ }^{4}$ A 30 anni pubblica i Principi di diritto amministrativo, la cui prima edizione è del $1890 .^{5}$ pubblico.

A 21 anni era già professore, e aveva fondato l'Archivio di diritto

Non giurò fedeltà al fascismo come professore, dimettendosi nel 1931 per dedicarsi solo all'attività forense.

Ben si comprende dunque che egli sia stato sempre considerato un Maestro. Egli stesso lo ammise pubblicamente: "non posso negare di esserlo; beninteso, però, senza la M maiuscola". ${ }^{6}$

1 Poi pubblicato, rivisto, e riferito alla riforma nel frattempo varata con la legge 22 gennaio 1882, n. 593, col titolo La riforma elettorale, Milano-Napoli-Pisa, Hoepli, 1883.

2 Composta dai commissari Carlo Cantoni, Cantù, Oliva, Vidari, Rajna relatore: cfr. i Rendiconti dell'Istituto, serie II, volume XIV, Milano-Napoli-Pisa, Hoepli, 1881, p. 742.

3 Ivi, p.748. In realtà Orlando nel 1879 aveva da poco intrapreso gli studi universitari, avendo 19 anni. Il passo citato della relazione della commissione è riprodotto anche nella prefazione allo studio pubblicato nel 1883 (La riforma, cit., pag. X, nota 2) in cui l'Autore ha cura di precisare che aveva compiuto i suoi studi nel 1881 (ivi, nota 3).

4 V.E. ORLANDO, Principi di diritto costituzionale, 1a ed., 1889.

5 V.E. ORLANDO, Principi di diritto amministrativo, $1^{\text {a }}$ ed., 1890.

6 Cfr. Atti Assemblea Costituente, seduta del 10 marzo 1947, pag. 1930. 


\section{ORLANDO PROTAGONISTA POLITICO DELl'ItALIA PREFASCISTA}

Ma Orlando attraversò due secoli da protagonista anche come uomo politico. La sua prima battaglia elettorale risale al 1895; eletto per la prima volta alla Camera nel 1897, fu parlamentare per 8 legislature del periodo prefascista, fino alle dimissioni nel 1925; Presidente del Consiglio (1917-19); Presidente della Camera (1919-20). Nel 1924 passò all'opposizione rispetto al Governo fascista e nel 1925 si dimise dal Parlamento.

Non fu, come si direbbe oggi, un tecnico prestato alla politica, ma fu un vero "professionista della politica". Non a caso si autodefinì ironicamente, nell'ultimo discorso che tenne nell'Assembla Costituente, "bomo parlamentaris per eccellenza" e già prima aveva detto: "tutta la mia vita è stata in funzione di parlamentarità"

Sempre sul rapporto fra il suo essere riconosciuto come maestro e il suo ruolo politico, usò queste parole: "Il maestro, così o comunque detto, in un'Assemblea sovrana, è assolutamente fuori posto ... Il professore, cioè il puro teorico, non è amato e bisogna dire, non di rado, con ragione in un'Assemblea che deve tener sempre i contatti con la viva realtà. Indubbiamente, io vengo qui come un tecnico che si sovrappone al politico (...) Dateci dei tecnici al Governo: ecco l'invocazione imperativa dell'uomo della strada". Ma "il tecnico della politica è l'uomo politico!". "Il vero uomo di Stato, nelle questioni tecniche che deve affrontare, deve sapere servirsi degli esperti o dei tecnici, ma deve poi tradurre le loro conoscenze in un'azione di Governo e politica, per cui occorre ben altra vocazione, ben altra intuizione e ben altra esperienza".

Dopo la parentesi fascista (per lui fu davvero una parentesi) Orlando affrontò una nuova stagione politica e istituzionale alla bella età di 83 anni!

Consultato da Vittorio Emanuele III quando si preparava il rivolgimento del 25 luglio 1943, si dice abbia contribuito a scrivere il testo del proclama Badoglio ("la guerra continua"): ma spiegò in seguito: "In generale, chi dà un consiglio per accondiscendere ad una richiesta, dovrebbe aspirare a questa garanzia minima: che non si cerchi, dopo gli

7 Atti cit., seduta del 22 dicembre 1947, pag. 3599.

8 Atti cit., seduta del 10 marzo 1947, pag. 1933.

9 Atti cit., seduta del 10 marzo 1947, pagg. 1930-31. 
eventi, di riversare sul consigliere una quota di responsabilità di un'azione cui questi non ha partecipato; il che vale tanto più, quando il dare un consiglio costituisce un dovere verso il rappresentante della sovranità dello Stato ... Comunque, i consigli che diedi non furono seguiti" ${ }^{10}$

Nel periodo pre-costituente, fu membro di diritto della Consulta in quanto Presidente del Consiglio prima del fascismo.

\section{Orlando alla Costituente: Il SUO PRIMO DisCORSO}

Eletto all'Assemblea Costituente con l'Unione democratica nazionale, che ottenne il 6,8\% dei voti, fu Presidente del gruppo, ma lo lasciò ben presto per passare al gruppo misto (si può forse pensare a qualche contrasto con Francesco Saverio Nitti?). Dell'Assemblea fu, in quanto decano, presidente provvisorio nella prima seduta del 25 giugno 1946, in cui fu eletto Presidente Saragat. Tenne il suo primo discorso nella seduta da lui presieduta come decano, e l'ultimo discorso nella seduta in cui fu approvata definitivamente la Costituzione, il 22 dicembre 1947.

Nel primo discorso, all'apertura dei lavori dell'Assemblea, la consapevolezza del particolare momento storico si salda con l'appello alla concordia nazionale.

"In quest'Assemblea - egli disse fra l'altro - (...) il popolo italiano è sovrano, ma, anche, il solo sovrano, l'arbitro assoluto della decisione del proprio destino. Sarebbe vana la ricerca se meglio convenga per il progresso politico e civile di un popolo il processo di evoluzione o di rivoluzione" E ancora: "Compito formidabile (...) di ricostruzione $a b$ imis cui codesta Assemblea dovrà accingersi, in un momento in cui nella eterna battaglia fra la libertà e la tirannide sembra che i popoli cerchino un ubi consistam fra il tramonto del Governo parlamentare e il delinearsi di un ordine nuovo in cui i partiti da semplici forze politiche verrebbero assumendo figura e caratteri di natura giuridica costituzionale, come organizzazioni delle masse sociali rappresentative del lavoro, considerando quest'ultimo come il fattore ormai assolutamente prevalente nella produzione e nella distribuzione della ricchezza”. Il limite in questa ricerca del nuovo ordine è la forma di governo repubblicana, onde il "dovere civico essenziale" è onorare e servire "questo simbolo ... come rappresentativo della Patria stessa, al di sopra e malgrado qual-

10 Atti cit., seduta del 30 luglio 1947, pag. 6498. 
siasi altra opinione o sentimento o ideale che si sia professato o che possa ancora essere professato". Ricordò la collaborazione, durante la prima grande guerra, di socialisti e repubblicani. Accennò alla pace "crudelmente punitiva" che si prospettava ("non intendiamo cadere nell'abisso di questa pace"), affermando: "Noi aspetteremo la nostra rivincita non in forma di una guerra, che ferventemente deprechiamo in nome della civiltà in pericolo; ma poiché ci si vuole distruggere, la nostra rivincita consisterà nella nostra risurrezione, nella quale abbiamo una fede fermissima". E concluse: "in questo pericolo mortale che ci minaccia dall'estero, un imperativo categorico si pone verso l'interno: l'unione, la pacificazione, la concordia ... la concordia nazionale perché si salvi l'Italia, perché viva l'Italia". ${ }^{11}$

\section{La POlEmica SUl Trattato Di PaCE}

La sua partecipazione ai dibattiti in Assemblea fu attiva anche se non molto frequente, ${ }^{12}$ ed egli fu sempre ascoltato con grande rispetto. Non mancarono anche suoi interventi assai vivaci e polemici. Celebre la sua "requisitoria" contro la ratifica del Trattato di pace, ad illustrazione di un ordine del giorno da lui presentato, inteso a rinviare l'approvazione del disegno di legge, e conclusa con una vera e propria "invettiva" che suscitò la reazione di De Gasperi e della DC: "Questi sono voti di cui si risponde dinanzi alle generazioni future; si risponde nei secoli di queste abiezioni fatte per cupidigia di servilità". Il resoconto annota, con singolare precisione, alla fine del discorso: "Vivissimi applausi a sinistra e a destra - Proteste vivaci al centro e al banco del Governo Rumori vivissimi - Scambio di epiteti fra sinistra e centro - Ripetuti richiami del Presidente - Nuovi prolungati applausi a sinistra e a destraProteste e rumori vivissimi al centro - Scambio di apostrofi fra il centro e le sinistre - Viva agitazione". ${ }^{13}$ Subito dopo Orlando, invitato dal Presidente dell'Assemblea a spiegarsi, affermò che "la parola «servilità» qualifica l'atto, e non le persone" e che il suo appello voleva essere "per la concordia, o almeno, contro l'esasperazione dei contrasti inevitabili”,

11 Atti cit., seduta del 25 giugno 1946, pagg. 1-3.

12 In proposito v. quanto egli stesso disse il 22 ottobre 1947: infra, nota 24.

13 Atti cit., seduta del 30 luglio 1947, pag. 6512. 
pur riaffermando che il Trattato di pace era "una solenne ingiustizia". La risposta di De Gasperi fu dura, volta a contestare l'accusa di "servilità" ("No! Onorevole Orlando: si tratta di concezione diversa degli interessi del Paese in questo momento") e a denunciare l'offesa a coloro che "hanno affrontato il nemico non soltanto come combattenti sui campi di battaglia, ma hanno affrontato il fascismo con coraggio, soffrendo giorno per giorno". ${ }^{14}$

L'incidente si chiuse in modo "cavalleresco": con De Gasperi che disse "avrei aspettato dalla sua lealtà che ella avesse dichiarato che le parole «cupidigia di servilità» non si riferivano a coloro che propongono in buona fede e con retta coscienza di ratificare il Trattato"; Orlando che replicò "L'ho detto", e De Gasperi che concluse: "se questa è la sua dichiarazione, io sono lieto di accettarla, quantunque il linguaggio altrimenti doveva essere interpretato". ${ }^{15} \mathrm{Il}$ "linguaggio parlamentare" allora aveva questi toni.

Si noti fra l'altro che si era in quel momento in una fase politica delicata e conflittuale, all'indomani della fine del governo tripartito DC-PSI-PCI (il terzo Governo De Gasperi, che vedeva ancora i tre partiti insieme): dal 31 maggio 1947 era in carica il IV Governo De Gasperi, sorretto dalla sola maggioranza centrista DC-PLI-PSLI-PRI.

\section{L'INTERVENTO DI ORLANDO NEL DIBATTITO GENERALE SUL PROGETTO DI COSTITUZIONE: SULLA FORMA DI GOVERNO}

Orlando, al pari degli altri maggiori esponenti della classe politica prefascista, rimasti estranei ai partiti di massa protagonisti della fase costituente, non fece parte della Commissione dei 75 , incaricata di elaborare il progetto di Costituzione, ma non mancò di prendere parte attivamente al dibattito in aula, anche chiedendone la necessaria estensione e perorando la proroga (poi deliberata con la legge cost. n. 2 del 17 giugno 1947) dei lavori dell'Assemblea. ${ }^{16}$

Sul testo del progetto di Costituzione, il suo ampio e denso inter-

14 Atti cit., pag. 6513.

15 Atti cit., pag. 6514.

16 Cfr. l'intervento di V. E. Orlando sugli ordini del giorno proposti sui metodi di lavoro dell'Assemblea: Atti cit., seduta del 2 maggio 1947, pagg. 3465-67. 
vento fu pronunciato nel dibattito generale del 10 marzo $1947 .{ }^{17}$ Disse in premessa di intervenire in quanto chiamato in causa più volte come "maestro", non potendo negare di esserlo. Criticò la posizione di coloro che invocano i "tecnici al Governo", perché - disse - "il tecnico della politica è l'uomo politico". ${ }^{18}$ Sul testo, espresse "approvazione", anzi "lode agli autori", osservando che la Commissione "ha fatto un vero miracolo", e lamentando la "assenza del Governo", che dovrebbe "essere sempre onnipresente in un'Assemblea"; ma al tempo stesso espresse sul testo "le più ampie riserve". E lamentò che si dovesse procedere "sotto l'assillo di questa urgenza". In generale, osservò che "le costituzioni le fanno assai più il costume, assai più la maniera della loro attuazione, anziché la fredda redazione degli articoli", ciò che "conforta, perché vuol dire che la soma si può accomodare per via", anche se "sconforta soprattutto coloro che sono animati dall'orgoglio (...) d'illudersi che basti la volontà dell'uomo per compiere l'atto creativo della maniera di essere, dell'ordinamento dello Stato di un popolo"; così che - concluse - "possiamo confidare che, se e in quanto delle deficienze si riscontrino nella Carta costituzionale che spetta a noi di redigere, l'esecuzione, col tempo, riesca a correggerle e ad integrarle". ${ }^{19}$

Entrando poi nel merito, iniziò dalla seconda parte, ritenendo che "per provvedere degnamente alle future sorti del Paese, sia quest'ultima che di gran lunga prevalga". In proposito osservò criticamente che il sistema che si voleva instaurare non era un vero sistema parlamentare, secondo l'originario modello inglese, anzitutto perché le funzioni legislativa ed esecutiva non erano assegnate ad "organi sovrani contrastanti", nel senso di "liberi da ogni gerarchia tra loro, equivalentisi, interferenti", in un sistema in cui "ognuno partecipa dell'altro in maniera da determinare una collaborazione e da impedire la sopraffazione". Nel progetto, invece, egli disse, "il potere esecutivo ora è il Capo dello Stato, ora il Governo; sfugge l'unità del funzione"; il Capo dello Stato (non più collocato come era "il Re in Parlamento") era escluso dall'attività legislativa, e non aveva il potere di sanzione delle leggi; l'iniziativa legislativa era ampiamente riconosciuta a molti soggetti ed organi, indebolendo così quella del Governo; mancava "questa compenetrazione

17 Atti cit., seduta del 10 marzo 1947, pagg. 1930-1944.

18 Cfr. supra, testo e nota 9.

19 Atti cit., seduta del 10 marzo 1947, pagg. 1930-32. 
reciproca, questo vivere insieme, collaborando e controllandosi", che "ha la sua espressione esterna, personificata, vivente" nel "Gabinetto", che, "da un lato, è potere esecutivo, deriva dal Capo dello Stato, lo rappresenta, e d'altro lato, è Parlamento, ne fa parte, lo dirige, lo controlla, lo guida". ${ }^{20}$

In particolare, riguardo al Parlamento, criticò il "compromesso" fra bicameralismo e monocameralismo che nel progetto si era espresso con la previsione di due Camere elettive (di cui il Senato però eletto per un terzo dai consigli regionali e per due terzi a suffragio universale e diretto) e inoltre dell'Assemblea Nazionale, formata dalle due Camere riunite, ma dotata di poteri più ampi di quelli dell'attuale Parlamento in seduta comune, in particolare titolare ultima del rapporto fiduciario col Governo. Sul punto, come si sa, la Costituente corresse poi significativamente il progetto. Secondo Orlando, sarebbe stata l'Assemblea Nazionale a detenere veramente il potere, ad avere "le chiavi della cassaforte". Di fatto essa avrebbe nominato e licenziato il Governo, mentre "nella genuina forma di Governo parlamentare" il Capo dello Stato, in caso di crisi, cerca di "trovare la soluzione più idonea" e alla fine "prende una decisione sotto la sua responsabilità, per quanto coperta dal nuovo Presidente del Consiglio". ${ }^{21}$

Il Capo dello Stato avrebbe avuto invece "la figura di un fainéant, di un fannullone"; e il principio della controfirma ministeriale necessaria per la validità di ogni atto del Capo dello Stato era bensì, secondo Orlando, principio "essenziale per la forma di governo parlamentare", ma col quale avrebbe dovuto concorrere l'altra "condizione non meno essenziale: che il Gabinetto ripeta la sua autorità dal Capo dello Stato, pur dovendo, di regola, avere la fiducia del Parlamento".

Il timore di Orlando, di fronte a ciò che a lui appariva come "un Capo dello Stato, esautorato; un'Assemblea sostanzialmente unica (...) la quale detiene effettivamente tutti i poteri, dispone di tutte le leve", era quello di un "totalitarismo di Assemblea", in cui il "vero detentore dell'autorità" sarebbe stato il Primo Ministro, che avrebbe avuto di fatto "tutti i poteri". Questi sarebbe stato "proprio un dittatore", se fosse stato "capo di un partito, che ha la maggioranza nell'Assemblea"; nel caso, che considerava più probabile, che continuasse "il sistema di

20 Atti cit., pagg.1932-35.

21 Atti cit., pagg. 1935-37. 
una coalizione di partiti", si sarebbe governato "attraverso accordi personali fra i capi dei partiti formanti la maggioranza", e quindi si sarebbe avuto "un Governo direttoriale" che "suppone una pluralità di capi non fusi nell'unità direttiva" ( $\mathrm{e}$ in proposito scherzosamente immaginò un triumvirato fra De Gasperi, Nenni e Togliatti, fra cui solo quest'ultimo avrebbe avuto "una linea da medaglia"). ${ }^{22}$

Il modello cui Orlando si rifaceva idealmente era quello storico di una Monarchia parlamentare che non avesse dismesso del tutto le caratteristiche, da cui si era sviluppata, della "Monarchia costituzionale", in cui il Re, lungi dall'essere escluso dal circuito dell'indirizzo politico, ne faceva pienamente parte, pur avendo dismesso la sua autorità di monarca assoluto, in nome del "patto" fra Re e popolo che caratterizzava le forme di governo nate in Europa con la Restaurazione, e di cui lo Statuto Albertino, che esplicitamente si poneva come costitutivo di un "Governo monarchico rappresentativo", era espressione. Non a caso, parlando il giorno successivo, Togliatti, pur affermando di trovare giuste alcune delle osservazioni di Orlando ("correggeremo, preciseremo"), disse di avere avuto l'impressione che Orlando cercasse nel progetto "qualcosa che noi non abbiamo voluto mettere nella Costituzione: che ella cercasse il re". ${ }^{23}$

Si collega a questo tema il significativo dibattito che ebbe luogo, alcuni mesi dopo, fra Orlando e Ruini, il Presidente della Commissione dei 75 .

In un intervento del 22 ottobre 1947, discutendosi sul potere di scioglimento delle Camere, Orlando disse che avrebbe voluto spiegare la sua "scarsa partecipazione ai lavori finora diretti alla creazione della nuova Costituzione e quella, temo, egualmente scarsa, ai lavori futuri", col suo dissenso "su quello che è lo spirito fondamentale, e non riguarda la differenza analitica su questa o su quella espressione" ("è come parlare due lingue diverse reciprocamente incomprese!"). ${ }^{24}$

Tornando su "uno dei punti più gravi" del suo dissenso, "la esautorazione completa" del Capo dello Stato e del potere esecutivo (tanto che avrebbe voluto "arrivare all'elezione popolare, appunto per rafforzarlo”), si oppose però al riconoscimento al Presidente di un potere di

22 Atti cit., pagg. 1937-39.

23 Atti cit., seduta pomeridiana dell'11 marzo 1947, pag. 1997.

24 Atti cit., seduta del 22 ottobre 1947, pagg. 1457-58. 
scioglimento come potere personale, "di prerogativa", sottratto al principio della responsabilità ministeriale, che sarebbe stato "radicalmente contrastante con lo spirito democratico". ${ }^{25}$

Il giorno successivo, ad inizio di seduta, intervenne Ruini, per fare "un breve cenno sulle osservazioni che la voce autorevolissima di Orlando ha fatto al nostro progetto di Costituzione". Riferendosi alla critica orlandiana alla "esautorazione" del Capo dello Stato, Ruini replicò sostenendo che in realtà si era previsto di conferire ad esso molte significative attribuzioni, facendone "una figura abbastanza forte e spiccata". Ma aggiunse che quello cui si voleva dare vita era ancora uno Stato parlamentare, ma "non è più precisamente il tipo storico caro all'onorevole Orlando. E' qualcosa di diverso, perché tutte le sue fondamenta sono ormai nella sovranità popolare". Tuttavia aggiunse Ruini - "non vogliamo che il Parlamento diventi tutto", andando incontro alla "concezione estremista e rivoluzionaria che vuol fare del Parlamento il vero e solo sovrano": si voleva ricorrere ad un sistema di "equilibri e di contrappesi ... fra i poteri che attingono alla fonte del popolo, esso sì sovrano". Ruini evocò i "tre reliquati" dello Statuto Albertino che la Costituente non poteva accogliere: il potere legislativo "collettivamente esercitato dal Re e dalle due Camere" (donde il rifiuto di attribuire al Presidente, insieme al potere di promulgazione, quello di "sanzione" delle leggi); l'appartenenza "al Re solo" del potere esecutivo (spettante invece al Governo, il cui capo non è il Presidente della Repubblica); la giustizia che "emana" dal Re ed è amministrata in suo nome dai giudici da lui istituiti (e che doveva invece essere esercitata in nome del popolo, con le garanzie di indipendenza per i magistrati). Onde, concluse Ruini, il progetto di Costituzione era ben lontano dalla concezione "ultraparlamentare" "degli onorevoli Togliatti e Nenni", ma anche "da quella troppo conservatrice degli onorevoli Orlando e Nitti", che era "una concezione ancora monarchica", e non si addiceva alla "democrazia repubblicana, che noi cerchiamo di fondare". ${ }^{26}$

Orlando chiese subito di intervenire per il "fatto personale, scientifico e giuridico" derivante dall'intervento di Ruini, e ne contestò vivacemente le tesi. Rivendicò di non essere "meno democratico in confron-

25 Atti cit., pagg. 1458-60.

26 Cfr. Atti cit., seduta del 23 ottobre 1947, pagg. 1465-68. 
to a voi, se voglio un Capo dello Stato repubblicano con più larga competenza e quindi maggiore autorità del Capo dello Stato monarchico"; affermò che le disposizioni dello Statuto, che Ruini aveva chiamato "reliquati", erano ormai "sorpassate dalla consuetudine (...) non esistevano più"; ribadì che il progetto aveva esautorato il Presidente della Repubblica "per ciò che riguarda competenza e attribuzioni nell'ordine del potere esecutivo"; che "non si capisce come questo Governo si colleghi col Presidente della Repubblica, se ne dipenda o sia autonomo"; che gli autori del progetto avevano "rotto proprio quell'unità, fra Parlamento, Governo e Capo dello Stato, che è la caratteristica di quella forma parlamentare, creata proprio in Inghilterra" ${ }^{27}$

\section{Sulla Corte costituzionale}

Su altre questioni, diverse da quella della forma di governo in senso stretto, l'attenzione di Orlando, e anche la penetrazione della sua analisi, nel discorso del 10 marzo 1947, si manifestano in misura minore.

Così, il suo pensiero sulla grande novità rappresentata dalla previsione della Corte costituzionale era del tutto svalutativo. Orlando sosteneva che sarebbero mancate totalmente in essa le condizioni, dalle quali può derivare l'autorità di un istituto, e cioè la forza politica che l'istituzione rappresenta o "la tradizione che si è venuta formando". La Corte infatti sarebbe stata formata per metà di magistrati (poi solo per un terzo nel testo finale), e questi, secondo la sua esperienza - diceva Orlando - anche se perfetti nell'esercizio delle loro funzioni, quando sono di fronte alla "sovranità dello Stato", quando è in gioco lo Stato, avevano "un istintivo movimento reverenziale" che turbava "il perfetto equilibrio del valutare la ragione ed il torto"; sono "un ordine il quale, per la natura stessa dell'ufficio che riveste, ha una sensibilità, che in generale è per esso un pregio, in quanto lo tiene lontano dalla politica, ma non lo rende atto per questo nuovo ufficio in cui il diritto non si disgiunge dalla politica": E quanto alle altre categorie di eleggibili (professori e avvocati), Orlando si limitò ad aggiungere: "Io, per esempio, sarei eleggibile, ma sento che sarei un pessimo giudice". ${ }^{28}$

27 Cfr. Atti cit., pagg. 1468-70.

28 Atti cit., seduta del 10 marzo 1947, pagg. 1939-40. 


\section{Sulla PRIMA PaRTE DELla Costituzione}

Quanto alla prima parte del progetto di Costituzione, nel suo intervento nel dibattito generale Orlando si limita a criticare l'abbondanza di "definizioni", come quella di famiglia quale "società naturale" (poi l'Assemblea aggiungerà "fondata sul matrimonio"); a perorare la limitazione delle dichiarazioni di principio ai "diritti veramente tradizionali - quelli di libertà, di eguaglianza, di fraternità"; ad appoggiare toto corde la parte nuova di proclamazione dei diritti "che riguarda l'uomo non come individuo, ma l'uomo come membro di questa società" (distanziandosi dalla visione roussoviana idealizzata dello "stato di natura"); ad appoggiare, in nome del principio di fraternità, l'aggiunta ai diritti del "dovere della società di provvedere" ai "mali sociali" creati dalla convivenza (la malattia, la disoccupazione, l'indigenza, la miseria, lo stesso vizio, lo stesso delitto), quindi a sostenere i diritti sociali (a questo punto il resoconto annota: "Vivi, generali applausi"): per soffermarsi poi sul tema dei Patti lateranensi e su quello delle Regioni. ${ }^{29}$

Il 22 marzo, in sede di votazione sull'attuale art. 1 della Costituzione ("L'Italia è una Repubblica democratica fondata sul lavoro"), fece una brevissima dichiarazione di voto annunciando che si sarebbe sempre astenuto su formulazioni contenenti delle definizioni. ${ }^{30}$

Il 23 aprile 1947, intervenendo ad illustrazione del suo ordine del giorno tendente a escludere dalla Costituzione l'intero titolo II della parte II (quello relativo a famiglia, salute e scuola), disse di fare una questione di metodo, per cui gli sembrava che "questa Costituzione, almeno nella sua prima parte", fosse "radicalmente sbagliata"; tornò a criticare le "definizioni" e le "promesse" costituzionali, arrivando a sostenere che fosse un errore dare "rigidità" alla Costituzione, ritenendo non credibile che un organo come la Corte costituzionale potesse "avere questa grande autorità di controllare il legislatore, che è sovrano, e di annullare le leggi"; e affermò, contrapponendo alle disposizioni del progetto di Costituzione la disciplina del codice civile allora in vigore, che le prime, "tecnicamente", erano "fatte apposta per rimettere in dubbio la certezza del diritto". ${ }^{31}$

29 Atti cit., pagg. 1940-42.

30 Atti cit., seduta pomeridiana del 22 marzo 1947, pag. 2381.

31 Atti cit., seduta del 23 aprile 1947, pagg. 3239-45. 


\section{Sui Patti Lateranensi}

Sul tema dei Patti Lateranensi Orlando si sofferma la prima volta, come si è detto, nel suo intervento nel dibattito generale. Rivendica di essere stato autore, per parte dello Stato, del "patto centrale dell'accordo e della pacificazione", cioè del Trattato che chiuse la questione romana, rievocandone la storia; consente pienamente al riferimento fatto ad essi nella Costituzione, solo avanzando il dubbio "tecnico" sull'includervi una rinuncia al diritto di denunciarli, ciò che gli sembrava "un limite della sovranità". ${ }^{32}$

Sul tema tornò in un successivo intervento del 18 marzo 1947. Discutendosi delle disposizioni generali del progetto, fra cui l'articolo sui rapporti fra lo Stato e la Chiesa, chiese la parola per fatto personale, rivendicando la "lealtà", la "fedeltà" con cui "l'Italietta del periodo prefascista" osservò la legge delle guarentigie, "senza che vi fosse alcun bisogno d'includerla in uno Statuto costituzionale"; e tornando a rievocare in dettaglio le vicende che avevano caratterizzato gli accordi per la soluzione della questione romana e che lo avevano visto protagonista. ${ }^{33}$

\section{Sulle Regioni}

L'ultimo argomento affrontato da Orlando nel dibattito generale è la questione delle Regioni: al cui proposito Orlando afferma che, non essendosi scelta la strada dello Stato federale al momento dell'unificazione nazionale, ciò creerebbe ora "una difficoltà, per sé stessa insuperabile: come ricostruiamo queste regioni", visto che il nesso con gli Stati preunitari non c'è più? Ma - osserva - "questo non vale per le isole, perché le isole, per la loro configurazione geografica, per la mentalità speciale dell'isolano, trovano in natura l'origine della loro aspirazione all'autonomia": onde "l'esperimento siciliano" potrebbe costituire "un banco di prova". Osserva ancora, in garbata polemica con Francesco Saverio Nitti, che l'affermato passivo con cui la Sicilia avrebbe gravato sul bilancio dello Stato (quello che oggi si chiamerebbe il "residuo fiscale" negativo) "non deriverebbe da eccesso di spese, ma da deficien-

32 Atti cit., seduta del 10 marzo 1947, pagg. 1941-42.

33 Atti cit., seduta pomeridiana del 18 marzo 1947, pagg. 2263-67. 
za di incassi". La conclusione è comunque che "la Sicilia non può fare a meno dell'Italia e l'Italia non può fare a meno della Sicilia". ${ }^{34}$

Il 13 giugno 1947, intervenendo nella discussione su di un controverso ordine del giorno Bonomi e altri in materia di Regioni (anche da lui avversato, e poi respinto dall'Assemblea), tendente a sancire in linea di principio alcune limitazioni ai poteri da attribuire alle Regioni, confermò la propria posizione di difesa dell'autonomia siciliana, dicendo che "per quanto si riferisce agli statuti già concessi, non solo alla Sicilia, ma anche alla Sardegna e ad altre Regioni d'Italia [le Regioni ad autonomia speciale] questi rappresentano degli impegni i quali non potrebbero essere violati". ${ }^{35}$ E della sua "sicilianità" aveva già fatto professione il 2 maggio 1947, intervenendo nel dibattito sulla strage, avvenuta il giorno prima, di Portella della Ginestra ("attraverso me parla il popolo della Sicilia"), dicendo che questo atto di assassinio politico "ci ferisce nel nostro onore: onore di Siciliani, onore di isolani", che erano fieri "di avere il primato (...) nell'abominare gli eccidi e gli assassini politici, vedendo in ciò "un segno di superiorità morale che ci compensava della nostra povertà". ${ }^{6}$

\section{L'INTERVENTO Di ORLANDO SUlLa CASSAZIONE UNICA}

Nella seduta del 27 novembre 1947 Orlando, intervenendo nel dibattito sulla unicità o pluralità delle Cassazioni, sostenne quest'ultima tesi. Per lui "il diritto lo fa il popolo" e "quanto più vasto è lo spazio, dove si alimentano le radici di questa creazione popolare per eccellenza, e quanto più queste radici si accostano al popolo, in tutti i suoi strati, in tutte le sue forme, in tutte le sue regioni, tanto meglio!". Ancora, rimarcò che la riforma unificatrice della Cassazione era stata del fascismo, ed era stata motivata dall'intento di un accentramento, mentre affermò - "una così fatta unificazione di pensiero giuridico è contro natura, perché ogni ambiente può foggiare a se stesso le proprie maniere di concezione giuridica, le quali poi, fondendosi, possono dar vita ad una figura nuova" ${ }^{37}$

34 Atti cit., seduta del 10 marzo 1947, pagg. 1942-44.

35 Atti cit., seduta del 13 giugno 1947, pag. 4779.

36 Atti cit., seduta del 2 maggio 1947, pagg. 3440-41.

37 Atti cit., seduta del 27 novembre 1947, pagg. 2582-85. 


\section{IL DISCORSO FINALE}

A conclusione della seduta in cui fu definitivamente approvato il testo della Costituzione, ebbe la parola nuovamente, per un discorso finale, il decano dell'Assemblea Orlando. Onore che - egli disse "indubbiamente (...) debbo al titolo della mia maggior vecchiezza. Ma, forse, nel caso presente più che il computo numerico degli anni, può valere a conferirmi questo titolo l'essere io rappresentante estremo delle tre generazioni che hanno fatto l'Italia". ${ }^{38}$

Affermato poi che "quest'Assemblea può esser fiera del lavoro compiuto, pur attraverso contrasti", e che "vi è solidarietà, unità, anche fra coloro che hanno sostenuto le tesi più diverse e più opposte, perché in ciò sta la bellezza della libertà parlamentare"; espressa la gratitudine dell'Assemblea al Capo provvisorio dello Stato Enrico De Nicola, che con la Costituzione assumeva il titolo di Presidente della Repubblica, e al Presidente della Costituente Umberto Terracini; ricordò come "così frequentemente e così manifestamente" egli avesse dichiarato una sua "diversità di pensare e di sentire" a proposito del testo costituzionale, osservando che "sono venute di fronte due diverse maniere di concepire l'intervento del legislatore nel fissare l'ordinamento giuridico di un popolo". Da un lato "l'imposizione di una regola attraverso una volontà consapevole", dall'altro lato l'idea, da lui sempre seguita, per cui "il diritto viene concepito non come una imposizione dall'esterno, ma come una qualche cosa di organico, che si sviluppa da sé". Espresse poi il suo "augurio fiducioso", ripetendo il motto a lui caro per cui "la soma si accomoda per via". Tornato a lodare "il dissenso, il contrasto come il mezzo più idoneo per scoprire la verità o per avvicinarsi ad essa il più che sia possibile", verità "naturalmente di un valore del tutto relativo", affermò: "Ma da questo momento tutto ciò è finito. Ora la Costituzione ha avuto la sua consacrazione laica. Essa è al di sopra delle sue discussioni". ${ }^{9}$

Osservò che un auspicio si poteva trarre dalla coincidenza dell'entrata in vigore della Costituzione con il centenario del 1848; e, chiedendosi "che cosa ci riserba il mondo", espresse la convinzione che questa "non è (...) una rivoluzione di ordinaria amministrazione"; è "un'era che succede ad un'altra; è un tipo di Stato che si sovrappone ad un altro". Allo Stato nazionale "si sostituirà una forma di superstato", anche se non si poteva

38 Atti cit., seduta del 22 dicembre 1947, pagg. 3598-99.

39 Atti cit., pagg. 3599-3600. 
dire (perché "non si fa l'indovino nella storia"), se a crearlo "sarà la forza o sarà l'accordo o sarà qualche cosa tra l'uno e l'altra", né se esso sarà "in un senso continentale" o "in un senso razziale". Di fronte a questo nuovo tipo di Stato che sorge "l'Italia è preparata a tutti i sacrifici, anche a quello della orgogliosa affermazione della sovranità assoluta", ma alla condizione che "questi limiti debbano valere pure per gli altri, per tutti gli altri" (parole in cui può scorgersi sia la nascente prospettiva europea, sia il consenso, sancito nell'articolo 11 della Carta, in condizioni di parità con gli altri Stati, alle "limitazioni di sovranità necessarie ad un ordinamento che assicuri la pace e la giustizia fra le nazioni"). Concluse affermando che "il nuovo sentimento, che potrà nascere, non sopprimerà l'antico", come nella storia è avvenuto con il passaggio dalle forme primitive di aggregazione ad altre sempre più ampie; onde non verranno mai meno i sentimenti di amore e di devozione verso "questa antica, gloriosa, veneranda Italia", che "dagli stessi contrasti potranno, anzi, esser resi più intensi". 40

\section{A MÒ DI CONCLUSIONE}

Rileggendo le parole di Orlando a settant'anni di distanza, non si può non rimanere colpiti, non tanto o non solo dalla lucidità di certe analisi e previsioni - non disgiunte da una certa connotazione di "conservatorismo", specie in tema di forma di governo e di garanzia costituzionale dei diritti, rispetto agli sviluppi del costituzionalismo contemporaneo - ma soprattutto dalla sua capacità - frutto anche della sua lunga esperienza politica - di scorgere e valutare i rapporti fra l'impianto formale delle norme e gli esiti e gli effetti che esse producono o potranno produrre sul piano sociale, politico e storico.

Ben si spiegano dunque, da un lato, l'unanime attenzione e il rispetto che tutte le parti politiche dimostrarono nei confronti suoi e delle sue tesi, dall'altro i rapporti di convergenza e di distanza emersi fra le concezioni costituzionali di cui egli era portatore e quelle delle forze politiche che hanno ispirato la Costituzione.

Rimane in ogni caso l'impronta nitida del pensiero e dell'azione di un protagonista eccezionale della vicenda costituzionale dell'Italia fra due secoli.

40 Atti cit., pagg. 3600-01. 